\title{
The Existence of Solutions for Boundary Value Problem of Fractional Functional Differential Equations with Delay
}

\author{
Mengrui Xu, Yanan Li, Yige Zhao and Shurong Sun ${ }^{a}$ \\ School of Mathematical, University of Jinan, Jinan, Shandong 250022, P R China
}

Abstract. A class of boundary value problem for fractional functional differential equation with delay $\left\{\begin{array}{l}{ }^{c} D^{\sigma} \omega(t)=f\left(t, \omega_{t}\right), t \in[0, \zeta], \\ \omega(0)=0, \omega^{\prime}(0)=0, \omega^{\prime \prime}(\zeta)=1,\end{array}\right.$ is studied, where $2<\sigma \leq 3, \quad{ }^{c} D^{\sigma}$ devote standard Caputo fractional derivative. In this article, three new criteria on existence and uniqueness of solution are obtained by Banach contraction mapping principle, Schauder fixed point theorem and nonlinear alternative theorem.

\section{Introduction}

In recent decades, fractional differential equations shows broad prospect owing to the wide application in science and engineering fields, for example, biology, chemical physics, control theory and viscoelastic mechanics [1-2]. Fractional-order calculus operators are theoretical generalization of integer order. In the past more than 300 years of expanding and growing of fractional calculus operators theory, numerous achievements were suggested by differ distinguished mathematicians, for example, Euler, Leibniz Griinwald and Weyl, from various perspectives. At present, there are many descriptions and formulations of fractional integral and differential operators. In this paper, we followed the Caputo fractional derivative and Riemann-Liouville fractional integral. Recently, boundary value problem (BVP) for fractional differential equation has aroused widespread concern. Most literatures have committed to ordinary fractional differential equations (e.g. [3-5]), fractional differential equation with delay is more useful, and fractional functional differential equation with delay is more accurate, but studies on fractional differential equations with delay have relatively little.

However, in many practical cases, time delay problems are constantly raised in various applied disciplines such as economics, pathology, meteorology and so on. Functional differential equations related to delay variables, so it has very wide application, and the study of it is of great practical significance. On the other hand, obviously its solution is more complicated than the classical ordinary one, that is to say, its solution theory is not as mature as the classical ordinary differential equation, which in turn affects the speed and breadth of its application. Although the complete basic theory

\footnotetext{
a Corresponding author: sshrong@163.com
}

remains to be established, it can be expected that this will be one of the hot topics in the future. Above all, fractional functional differential equation with delay is more accurate, however, at present, studies on fractional differential equations with delay have relatively little and there are many exploratory works on these new types of functional differential equations, so it has the significant theoretical and practical significance.

Recently, there are many papers about the fractional differential equations.

In 2017, Zhou et al. [4] has studied the fractional neutral functional differential equation. Some results on existence of nonoscillatory solutions are presented.

In 2018, Ravi P. Agarwal et al. [5] has addressed the multi-term fractional order nonlocal integral boundary value problems. Some criteria on existence of solutions are presented by means of standard tool.

However, have relatively little study has been done for BVP of fractional delay functional differential equation.

Motivated by the above, we are interested in fractional delay functional differential equation with boundary value conditions:

$$
\left\{\begin{array}{c}
{ }^{C} D^{\sigma} \omega(t)=f\left(t, \omega_{t}\right), t \in[0, \zeta]:=\Omega, \\
\omega(0)=0, \omega^{\prime}(0)=0, \omega^{\prime \prime}(\zeta)=1,
\end{array}\right.
$$

where $2<\sigma \leq 3, \quad{ }^{C} D^{\sigma}$ devote standard Caputo fractional derivative, the function $f: \Omega \times C(\Lambda) \rightarrow R, \Lambda=:[-r, 0], 0 \leq r<\zeta, C(\Lambda)$ is the Banach space equipped with $\|\omega\|_{J}=\max \{|\omega(t)|: t \in \Lambda\}$, and the $\omega_{t}$ devote $\omega_{t}(\theta)=\omega(t+\theta), \theta \in \Lambda$. 
The results on the uniqueness of solution for (1) are obtained take advantage of the Banach contraction mapping principle. The existence conclusions for (1) are concluded from Schauder fixed point theory, nonlinear alternative theorem, respectively. These main conclusions are show in Section 3. In section 2, the definitions and lemmas used in this article are presented. Finally, we shall give three examples.

\section{Preliminaries}

For the details of development, basic definitions and properties of fractional calculus operators, see the monograph [1-2].

Definition 1. ([1]) A function $\zeta$ with its $\delta$ derivative existing on the set $I$ is known as a solution of (1), if $\zeta$ meets the boundary value problem (1).

$C(\Omega \bigcup \Lambda)$ denotes the Banach space equipped with

$$
\|v\|_{\Omega \cup \Lambda}=\max \left\{\|v\|_{\Omega},\|v\|_{\Lambda}\right\}
$$

Cone $B \subset C(\Omega \bigcup \Lambda)$ is defined as

$$
B=\{v \in C(\Omega \bigcup \Lambda) \mid v(t)>0\}
$$

and set

$$
B_{\eta}=\left\{v \in B\|\mid v(t)\|_{\Omega \cup \Lambda} \leq \eta, \eta>0\right\}
$$

Lemma 1. Let $f: \Omega \times C(\Lambda) \rightarrow R$ be continuous. A function $\varpi$ is a solution of the integral equation

$$
\varpi(t)=\left\{\begin{array}{c}
\frac{t^{2}}{2}-\frac{t^{2}}{2 \Gamma(\sigma-2)} \int_{0}^{\zeta}(\zeta-s)^{\sigma-3} f\left(s, \varpi_{s}\right) d s \\
+\frac{1}{\Gamma(\sigma)} \int_{0}^{t}(t-s)^{\sigma-1} f\left(s, \varpi_{s}\right) d s, t \in \Omega, \\
\frac{t^{2}}{2}-\frac{t^{2}}{2 \Gamma(\sigma-2)} \int_{0}^{\zeta}(\zeta-s)^{\sigma-3} f\left(s, \varpi_{s}\right) d s, t \in \Lambda,
\end{array}\right.
$$

Proof. Applying $I^{\sigma}$ to equation (1), one has

$$
I^{\sigma C} D^{\sigma} \varpi(t)=I^{\sigma} f\left(t, \varpi_{t}\right), 2<\sigma \leq 3 .
$$

Then by the use of properties of fractional derivatives, we have

$$
\varpi(t)-C_{0}-C_{1} t-C_{2} t^{2}=\frac{1}{\Gamma(\sigma)} \int_{0}^{t}(t-s)^{\sigma-1} f\left(s, \varpi_{s}\right) d s,
$$

so

$$
\varpi(t)=\frac{1}{\Gamma(\sigma)} \int_{0}^{t}(t-s)^{\sigma-1} f\left(s, \varpi_{s}\right) d s+C_{0}+C_{1} t+C_{2} t^{2} .
$$

From the boundary value condition $\varpi(0)=0$ of (1), we have $C_{0}=0$. It is obvous that,

$$
\varpi^{\prime}(t)=\frac{1}{\Gamma(\sigma-1)} \int_{0}^{t}(t-s)^{\sigma-2} f\left(s, \varpi_{s}\right) d s+C_{1}+C_{2} t .
$$

Combining with $\varpi^{\prime}(0)=0$, one has $C_{1}=0$. By applying with $\varpi^{\prime \prime}(\zeta)=1$, we have

$$
\varpi^{\prime \prime}(\zeta)=2 C_{2}+\frac{1}{\Gamma(\sigma-2)} \int_{0}^{\zeta}(\zeta-s)^{\sigma-3} f\left(s, \varpi_{s}\right) d s=1,
$$

That is to say,

$$
2 C_{2}=1-\frac{1}{\Gamma(\sigma-2)} \int_{0}^{\zeta}(\zeta-s)^{\sigma-3} f\left(s, \varpi_{s}\right) d s .
$$

Therefore,

$$
\varpi(t)=\left\{\begin{array}{c}
\frac{t^{2}}{2}\left[1-\frac{1}{\Gamma(\sigma-2)} \int_{0}^{\zeta}(\zeta-s)^{\sigma-3} f\left(s, \varpi_{s}\right) d s\right] \\
+\frac{1}{\Gamma(\sigma)} \int_{0}^{t}(t-s)^{\sigma-1} f\left(s, \varpi_{s}\right) d s, t \in \Omega, \\
\frac{t^{2}}{2}\left[1-\frac{1}{\Gamma(\sigma-2)} \int_{0}^{\zeta}(\zeta-s)^{\sigma-3} f\left(s, \varpi_{s}\right) d s\right], t \in \Lambda,
\end{array}\right.
$$

is a solution of (1).

\section{Main results}

In the following section, taking advantage of the Banach contraction mapping principle the uniqueness conclusions for (1) are proved, by Schauder fixed point theory one of existence conclusions for (1) are obtained, and through nonlinear alternative of Leray-Schauder type some other new sufficient conditions are established.

Theorem 1. Suppose that

$\left(A_{1}\right)$ there is a constant $L>0$ satisfying

$$
\begin{gathered}
\left|f\left(t, \psi_{t}\right)-f\left(t, \ell_{t}\right)\right| \leq L|\psi-\ell|, t \in \Omega \bigcup \Lambda, \\
\psi, \ell \in B_{\eta} .
\end{gathered}
$$

If

$$
L \zeta^{\delta}\left[\frac{1}{\Gamma(\sigma+1)}+\frac{1}{2 \Gamma(\sigma-1)}\right]<1,
$$

Then the BVP (1) has a unique solution.

Proof. Defined operator $F: B_{\eta} \rightarrow C(\Omega \bigcup \Lambda)$ by

$$
F v(t)=\left\{\begin{array}{c}
\frac{t^{2}}{2}\left[1-\frac{1}{\Gamma(\sigma-2)} \int_{0}^{\zeta}(\zeta-s)^{\sigma-3} f\left(s, v_{s}\right) d s\right] \\
+\frac{1}{\Gamma(\sigma)} \int_{0}^{t}(t-s)^{\sigma-1} f\left(s, v_{s}\right) d s, t \in \Omega, \\
\frac{t^{2}}{2}\left[1-\frac{1}{\Gamma(\sigma-2)} \int_{0}^{\zeta}(\zeta-s)^{\sigma-3} f\left(s, v_{s}\right) d s\right], t \in \Lambda,
\end{array}\right.
$$

Let $\psi, \ell \in B_{\eta}$, for each $t \in I$, 


$$
\begin{gathered}
|F \psi(t)-F \ell(t)| \\
\leq \frac{1}{\Gamma(\sigma)} \int_{0}^{t}(t-s)^{\sigma-1}\left|f\left(s, \psi_{s}\right)-f\left(s, \ell_{s}\right)\right| d s \\
+\frac{\zeta^{2}}{2 \Gamma(\sigma-2)} \int_{0}^{\zeta}(\varsigma-s)^{\sigma-3}\left|f\left(s, \psi_{s}\right)-f\left(s, \ell_{s}\right)\right| d s \\
\leq \frac{L\|\psi-\ell\|_{\Omega \cup \Lambda}}{\Gamma(\sigma)} \int_{0}^{t}(t-s)^{\sigma-1} d s \\
+\frac{\zeta^{2} L\|\psi-\ell\|_{\Omega \cup \Lambda}}{2 \Gamma(\sigma-2)} \int_{0}^{\zeta}(\zeta-s)^{\sigma-3} d s \\
\leq L \zeta^{\delta}\left[\frac{1}{\Gamma(\sigma+1)}+\frac{1}{2 \Gamma(\sigma-1)}\right]\|\psi-\ell\|_{\Omega \cup \Lambda} .
\end{gathered}
$$

If $t \in J$,

$$
\begin{gathered}
|F \psi(t)-F \ell(t)| \\
\leq \frac{\zeta^{2}}{2 \Gamma(\sigma-2)} \int_{0}^{\zeta}(\zeta-s)^{\sigma-3}\left|f\left(s, \psi_{s}\right)-f\left(s, \ell_{s}\right)\right| d s \\
\leq \frac{L \zeta^{\sigma}}{2 \Gamma(\sigma-1)}\|\psi-\ell\|_{\Omega \cup \Lambda} .
\end{gathered}
$$

Therefore,

$$
\begin{gathered}
\|F \psi(t)-F \ell(t)\|_{\Omega \cup \Lambda} \\
\leq L \zeta^{\sigma}\left[\frac{1}{\Gamma(\sigma+1)}+\frac{1}{2 \Gamma(\sigma-1)}\right]\|\psi-\ell\|_{\Omega \cup \Lambda} .
\end{gathered}
$$

Thus, $F$ is contracting. According to Banach contraction principle, BVP (1) has a unique solution.

Theorem 2. Suppose that

$$
\left(A_{2}\right) f \in C(\Omega \times \Lambda, R) \text {; }
$$

$\left(A_{3}\right)$ there is a constant $\vartheta>0$ satisfying

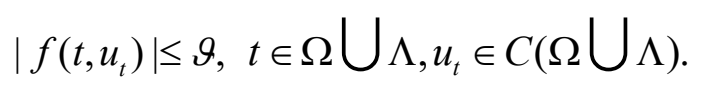

Then the BVP (1) has at least one solution.

Proof. Firstly, we shall prove $F$ (defined by (4)) is continuous. Let $\left\{\varpi_{n}^{*}\right\}$ be a sequence with $\varpi_{n}^{*} \rightarrow \varpi$ in $C\left(\Omega \bigcup_{\Lambda}\right)$. Then for each $t \in \Omega$,

$$
\begin{gathered}
\left|F \varpi_{n}^{*}(t)-F \varpi(t)\right| \\
\leq \frac{\zeta^{2}}{2 \Gamma(\sigma-2)} \int_{0}^{\zeta}(\zeta-s)^{\sigma-3}\left|f\left(s,\left(\varpi_{n}^{*}\right)_{s}\right)-f\left(s, \varpi_{s}\right)\right| d s .
\end{gathered}
$$

By continuity of $f$, one has

$$
\left\|F \varpi_{n}^{*}-F \varpi\right\|_{\Omega \cup \Lambda} \rightarrow 0, \text { as } n \rightarrow \infty .
$$

Secondly, clearly, for any $\eta>0$,

$B_{\eta}=\left\{v \in C(\Omega \bigcup \Lambda):\|v\|_{\Omega \cup \Lambda} \leq \eta\right\}$, there is a positive constant $l$ satisfying $\|F v\|_{\Omega \cup \Lambda} \leq l$. From $\left(A_{3}\right)$, for each $t \in \Omega$, we can get

$$
\begin{gathered}
|F v(t)| \leq \frac{\zeta^{2}}{2}\left[1+\frac{1}{\Gamma(\sigma-2)} \int_{0}^{\zeta} \frac{\left|f\left(s, v_{s}\right)\right|}{(\zeta-s)^{3-\sigma}} d s\right] \\
+\frac{1}{\Gamma(\sigma)} \int_{0}^{t}(t-s)^{\sigma-1}\left|f\left(s, v_{s}\right)\right| d s \\
\leq \frac{\zeta^{2}}{2}\left[1+\frac{\vartheta}{\Gamma(\sigma-2)} \int_{0}^{\zeta}(\zeta-s)^{\delta-3} d s\right]+\frac{\vartheta}{\Gamma(\sigma)} \int_{0}^{t}(t-s)^{\delta-1} d s \\
\leq \frac{\zeta^{2}}{2}+\frac{\vartheta \zeta^{\delta}}{\Gamma(\sigma+1)}+\frac{\vartheta \zeta^{\delta}}{2 \Gamma(\sigma-1)} .
\end{gathered}
$$

If $t \in \Lambda$,

$$
\begin{gathered}
|F v(t)| \leq \frac{\zeta^{2}}{2}\left[1+\frac{1}{\Gamma(\sigma-2)} \int_{0}^{\zeta}(\zeta-s)^{\sigma-3}\left|f\left(s, v_{s}\right)\right| d s\right] \\
\leq \frac{\zeta^{2}}{2}\left[1+\frac{\vartheta}{\Gamma(\sigma-2)} \int_{0}^{\zeta}(\zeta-s)^{\sigma-3} d s\right] \\
\leq \frac{\zeta^{2}}{2}+\frac{\vartheta \zeta^{\delta}}{2 \Gamma(\sigma-1)} .
\end{gathered}
$$

Thus,

$$
\|F v\|_{\Omega \cup \Lambda} \leq \frac{\zeta^{2}}{2}+\frac{\vartheta \zeta^{\sigma}}{\Gamma(\sigma+1)}+\frac{\vartheta \zeta^{\sigma}}{2 \Gamma(\sigma-1)}=l .
$$

Thirdly, we will show that $F$ is uniformly continuous.

Case 1. $\varpi \in B_{\eta}, \tau_{1}, \tau_{2} \in \Omega$ and $\tau_{1}<\tau_{2}$

$$
\begin{gathered}
\left|F \varpi\left(\tau_{2}\right)-F \varpi\left(\tau_{1}\right)\right|=\frac{\left(\tau_{2}-\tau_{1}\right)^{2}}{2} \\
+\mid \frac{1}{\Gamma(\sigma)} \int_{0}^{\tau_{1}}\left[\left(t^{2}-s\right)^{\sigma-1}-\left(\tau_{1}-s\right)^{\sigma-1}\right] f\left(s, \varpi_{s}\right) d s \\
+\frac{1}{\Gamma(\sigma)} \int_{\tau_{1}}^{\tau_{2}}\left(\tau_{2}-s\right)^{\sigma-1} f\left(s, \varpi_{s}\right) d s \\
+\frac{\tau_{1}^{2}-\tau_{2}^{2}}{2 \Gamma(\sigma-2)} \int_{0}^{\zeta}(\zeta-s)^{\sigma-3} f\left(s, \varpi_{s}\right) d s \mid \\
\leq \frac{\left(\tau_{2}-\tau_{1}\right)^{2}}{2}+\frac{\vartheta}{\Gamma(\sigma)} \int_{0}^{\tau_{1}}\left[\left(\tau_{2}-s\right)^{\sigma-1}-\left(\tau_{1}-s\right)^{\sigma-1}\right] d s
\end{gathered}
$$




$$
\begin{aligned}
+\frac{\vartheta}{\Gamma(\sigma)} \int_{\tau_{1}}^{\tau_{2}}\left(\tau_{2}-s\right)^{\sigma-1} d s+\frac{\vartheta\left(\tau_{2}-\tau_{1}\right)^{2}}{2 \Gamma(\sigma-2)} \int_{0}^{\zeta}(\zeta-s)^{\sigma-3} d s \\
\leq \frac{\left(\tau_{2}-\tau_{1}\right)^{2}}{2}+\frac{\vartheta}{\Gamma(\sigma+1)}\left[2\left(\tau_{2}-\tau_{1}\right)^{\sigma}+\tau_{1}{ }^{\sigma}-\tau_{2}{ }^{\sigma}\right] \\
+\frac{\vartheta\left(\tau_{2}-\tau_{1}\right)^{2}}{2 \Gamma(\sigma-1)} \zeta^{\delta-2} .
\end{aligned}
$$

Case 2. $\varpi \in B_{\eta}, \tau_{1}, \tau_{2} \in \Lambda$ and $\tau_{1}<\tau_{2}$,

$$
\begin{gathered}
\left|F \varpi\left(\tau_{2}\right)-F \varpi\left(\tau_{1}\right)\right| \\
=\frac{\left(\tau_{2}-\tau_{1}\right)^{2}}{2}\left|1+\frac{1}{\Gamma(\sigma-2)} \int_{0}^{\zeta}(\zeta-s)^{\sigma-3} f\left(s, \varpi_{s}\right) d s\right| \\
\leq \frac{\left(\tau_{2}-\tau_{1}\right)^{2}}{2}\left(1+\frac{\vartheta}{\Gamma(\sigma-2)} \int_{0}^{\zeta}(\zeta-s)^{\sigma-3} d s\right) \\
\leq \frac{\left(\tau_{2}-\tau_{1}\right)^{2}}{2}\left(1+\frac{\vartheta \zeta^{\sigma-2}}{\Gamma(\sigma-1)}\right) .
\end{gathered}
$$

Case 3. $\varpi \in B_{\eta},-r \leq \tau_{1}<0<\tau_{2} \leq \zeta$ :

$$
\begin{gathered}
\left|F \varpi\left(\tau_{2}\right)-F \varpi\left(\tau_{1}\right)\right| \\
\leq\left|F \varpi\left(\tau_{2}\right)-F \varpi(0)\right|+\left|F \varpi(0)-F \varpi\left(\tau_{1}\right)\right| \\
\leq \frac{\left(\tau_{2}-\tau_{1}\right)^{2}}{2}+\frac{1}{\Gamma(\sigma)} \int_{0}^{\tau_{2}}\left(\tau_{2}-s\right)^{\sigma-1}\left|f\left(s, \varpi_{s}\right)\right| d s \\
+\frac{\tau_{2}^{2}+\tau_{1}^{2}}{2 \Gamma(\sigma-2)} \int_{0}^{\zeta}(\zeta-s)^{\sigma-3}\left|f\left(s, \varpi_{s}\right)\right| d s \\
\leq \frac{\left(\tau_{2}-\tau_{1}\right)^{2}}{2}\left(1+\frac{\vartheta \zeta^{\sigma-2}}{\Gamma(\sigma-1)}\right)+\frac{\vartheta \tau_{2}^{\delta}}{\Gamma(\sigma+1)} \\
\leq \frac{\left(\tau_{2}-\tau_{1}\right)^{2}}{2}\left(1+\frac{\vartheta \zeta^{\sigma-2}}{\Gamma(\sigma-1)}\right)+\frac{\vartheta\left(\tau_{2}-\tau_{1}\right)^{\sigma}}{\Gamma(\sigma+1)} .
\end{gathered}
$$

Thus, $F B_{\eta}$ is equicontinuous. Taking advantage of Arzela-Ascoli theorem, $F: B_{\eta} \rightarrow B$ is completely continuous.

Finally, we shall prove the set

$Z=\{\varpi \in C(\Omega \bigcup \Lambda): \varpi=\eta F \varpi\}$ is bounded. If $\varpi \in Z$. Then $\varpi=\eta F \varpi$ for some $0<\eta<1$. Hence, for each $t \in \Omega$ we have

$$
\varpi(t)=\frac{\eta}{2}\left[\zeta^{2}-\frac{t^{2}}{\Gamma(\sigma-2)} \int_{0}^{\zeta}(\zeta-s)^{\sigma-3} f\left(s, \varpi_{s}\right) d s\right]
$$

$$
+\frac{\eta}{\Gamma(\sigma)} \int_{0}^{t}(t-s)^{\sigma-1} f\left(s, \varpi_{s}\right) d s
$$

by $\left(A_{3}\right)$, we can get

$$
\begin{aligned}
|v(t)| \leq & \frac{\zeta^{2}}{2}\left[1+\frac{\vartheta}{\Gamma(\sigma-2)} \int_{0}^{\zeta}(\zeta-s)^{\sigma-3} d s\right] \\
& +\frac{\vartheta}{\Gamma(\sigma)} \int_{0}^{t}(t-s)^{\sigma-1} d s \\
\leq \frac{\zeta^{2}}{2}+ & \frac{\vartheta \zeta^{\sigma}}{\Gamma(\sigma+1)}+\frac{\vartheta \zeta^{\sigma}}{2 \Gamma(\sigma-1)}, \quad t \in \Omega .
\end{aligned}
$$

Similarly, when $t \in \Omega$, we have

$\varpi(t) \leq \frac{\eta}{2}\left[\zeta^{2}+\frac{t^{2}}{\Gamma(\sigma-2)} \int_{0}^{\zeta}(\zeta-s)^{\sigma-3} f\left(s, \varpi_{s}\right) d s\right]$,

From $\left(A_{3}\right)$, one has

$$
|\varpi(t)| \leq \frac{\zeta^{2}}{2}\left[1+\frac{\vartheta}{\Gamma(\sigma-2)} \int_{0}^{\zeta}(\zeta-s)^{\sigma-3} d s\right]
$$

$$
\leq \frac{\zeta^{2}}{2}+\frac{\vartheta \zeta^{\sigma}}{2 \Gamma(\sigma-1)}, t \in \Lambda
$$

Therefore,

$$
\|\varpi\|_{\Omega \cup \Lambda} \leq \frac{\zeta^{2}}{2}+\frac{\vartheta \zeta^{\sigma}}{\Gamma(\sigma+1)}+\frac{\vartheta \zeta^{\sigma}}{2 \Gamma(\sigma-1)}, t \in \Omega \bigcup_{\Lambda} .
$$

Hence, $Z$ is bounded. Based on the Schauder fixed point theorem, BVP (1) has at least one solution.

Theorem 3. Let $\left(A_{2}\right)$ hold. Suppose that

$\left(A_{4}\right)$ there is a continuous non-decreasing function $\xi: R^{+} \rightarrow R^{+}$satisfying $f\left(t, \varpi_{t}\right) \leq \xi\left(\|\varpi\|_{\Omega \cup \Lambda}\right)$;

$\left(A_{5}\right)$ there is a constant $\gamma>0$ satisfying

$$
\frac{\gamma}{\frac{\zeta^{2}}{2}+\frac{\xi(\gamma) \zeta^{\sigma}}{\Gamma(\sigma+1)}+\frac{\xi(\gamma) \zeta^{\sigma}}{2 \Gamma(\sigma-1)}}>1
$$

Then the BVP (1) has at least one solution.

Proof. Let $\varpi$ such that $\varpi=\eta F \varpi$ for some $0<\eta<1$. Then

$$
\varpi(t)=\eta\left\{\begin{array}{c}
\frac{t^{2}}{2}\left[1-\frac{1}{\Gamma(\sigma-2)} \int_{0}^{\zeta}(\zeta-s)^{\sigma-3} f\left(s, \varpi_{s}\right) d s\right] \\
+\frac{1}{\Gamma(\sigma)} \int_{0}^{t}(t-s)^{\sigma-1} f\left(s, \varpi_{s}\right) d s, t \in \Omega, \\
\frac{t^{2}}{2}\left[1-\frac{1}{\Gamma(\sigma-2)} \int_{0}^{\zeta}(\zeta-s)^{\sigma-3} f\left(s, \varpi_{s}\right) d s\right], t \in \Lambda .
\end{array}\right.
$$


By $\left(A_{4}\right)$, for $t \in \Omega$, one has

$$
\begin{aligned}
|\varpi(t)|= & \eta \mid \frac{1}{2} t^{2}\left(1-\frac{1}{\Gamma(\sigma-2)} \int_{0}^{\zeta}(\zeta-s)^{\sigma-3} f\left(s, \varpi_{s}\right) d s\right. \\
& +\frac{1}{\Gamma(\sigma)} \int_{0}^{t}(t-s)^{\sigma-1} f\left(s, \varpi_{s}\right) d s \mid \\
\leq & \frac{\zeta^{2}}{2}\left[1+\frac{\xi\left(\|\varpi\|_{\Omega \cup \Lambda}\right)}{\Gamma(\sigma-2)} \int_{0}^{\zeta}(\zeta-s)^{\sigma-3} d s\right] \\
& +\frac{\xi\left(\|\varpi\|_{\Omega \cup \Lambda}\right)}{\Gamma(\sigma)} \int_{0}^{t}(t-s)^{\delta-1} d s \\
= & \frac{\zeta^{2}}{2}+\frac{\xi\left(\|\varpi\|_{\Omega \cup \Lambda}\right) \zeta^{\sigma}}{\Gamma(\sigma+1)}+\frac{\xi\left(\|\varpi\|_{\Omega \cup \Lambda}\right) \zeta^{\sigma}}{2 \Gamma(\sigma-1)} .
\end{aligned}
$$

When $t \in \Lambda$, one has

$$
\begin{gathered}
|\varpi(t)|=\eta \mid \frac{1}{2} t^{2}\left(1-\frac{1}{\Gamma(\sigma-2)} \int_{0}^{\zeta}(\zeta-s)^{\sigma-3} f\left(s, \varpi_{s}\right) d s \mid\right. \\
\leq \frac{\zeta^{2}}{2}\left[1+\frac{\xi\left(\|\varpi\|_{\Omega \cup \Lambda}\right)}{\Gamma(\sigma-2)} \int_{0}^{\zeta}(\zeta-s)^{\sigma-3} d s\right] \\
\leq \frac{1}{2} \zeta^{2}+\frac{\xi\left(\|\varpi\|_{\Omega \cup \Lambda}\right) \zeta^{\sigma}}{2 \Gamma(\sigma-1)} .
\end{gathered}
$$

That is to say,

$$
|\varpi(t)| \leq \frac{\zeta^{2}}{2}+\frac{\xi\left(\|\varpi\|_{\Omega \cup \Lambda}\right) \zeta^{\sigma}}{\Gamma(\sigma+1)}+\frac{\xi\left(\|\varpi\|_{\Omega \cup \Lambda}\right) \zeta^{\sigma}}{2 \Gamma(\sigma-1)}, t \in \Omega \bigcup_{\Lambda} .
$$

Therefore,

$$
\frac{\|\varpi\|_{\Omega \cup \Lambda}}{\frac{\zeta^{2}}{2}+\frac{\xi\left(\|\varpi\|_{\Omega \cup \Lambda}\right) \zeta^{\sigma}}{\Gamma(\sigma+1)}+\frac{\xi\left(\|\varpi\|_{\Omega \cup \Lambda}\right) \zeta^{\sigma}}{2 \Gamma(\sigma-1)}} \leq 1 .
$$

In view of $\left(A_{5}\right)$, there is a constant $\gamma$ satisfying $\|\varpi\|_{\Omega \cup \Lambda} \neq \gamma$. Set $G=\{\varpi \in C(\Omega \bigcup \Lambda):\|\varpi\|<\gamma\}$. It is obvious that there is no $\mu \in \partial G$ satisfying $\varpi=\eta F \varpi$ for $0<\eta<1$. On the other hand, we can see operator $F: \bar{G} \rightarrow C(\Omega \bigcup \Lambda)$ is completely continuous from the proof of Theorem 2. Taking advantage of the Leray-Schauder nonlinear alternative, BVP (1) has at least one solution.

Example 3.1 Consider the BVP of $\delta$-order functional differential equations with delay:

$$
\begin{gathered}
{ }^{C} D^{\sigma} \varpi(t)=\frac{\left|\varpi_{t}\right|}{\left(3+4 e^{t}\right)\left(2+\left|\varpi_{t}\right|\right)}, \\
\varpi(0)=0, \varpi^{\prime}(0)=0, \varpi^{\prime \prime}(1)=1,
\end{gathered}
$$

where $t \in \Omega \bigcup \Lambda=[0,1] \bigcup_{[-1,0], 2<\sigma \leq 3 \text {. Set }}$

$$
f(t, \varpi)=\frac{|\varpi|}{\left(3+4 e^{t}\right)(2+|\varpi|)} .
$$

Let $\varpi, \omega \in C[-1,1]$. Then for each $t \in \Lambda$,

$$
\begin{gathered}
|f(t, \varpi)-f(t, \omega)| \leq \frac{1}{3+4 e^{t}}\left(\frac{|\varpi|}{2+|\varpi|}-\frac{|\omega|}{2+|\omega|}\right) \\
=\frac{2(|\varpi|-|\omega|)}{\left(3+4 e^{t}\right)(2+|\varpi|)(2+|\omega|)} \leq \frac{1}{2\left(3+4 e^{t}\right)}|\varpi-\omega| \\
\leq \frac{1}{14}|\varpi-\omega| .
\end{gathered}
$$

For each $t \in \Lambda$,

$$
\begin{gathered}
|f(t, \varpi)-f(t, \omega)| \leq \frac{2|\varpi-\omega|}{\left(3+4 e^{t}\right)(2+|\varpi|)(2+|\omega|)} \\
\quad \leq \frac{1}{2\left(3+\frac{4}{e}\right)}|\varpi-\omega| \approx 0.1118|\varpi-\omega| .
\end{gathered}
$$

We can find $\zeta=1, L=\left[2\left(3+\frac{4}{e}\right)\right]^{-1} \approx 0.1118$. Thus the $\left(A_{1}\right)$ is satisfied. If $L \zeta^{\sigma}\left[\frac{1}{\Gamma(\sigma+1)}+\frac{1}{2 \Gamma(\sigma-1)}\right]<1$, that is to say, $\frac{1}{\Gamma(\sigma+1)}+\frac{1}{2 \Gamma(\sigma-1)}<2\left(3+\frac{4}{e}\right)$, then, applying Theorem 1 , for some $\sigma \in(2,3]$ satisfying

$$
\frac{1}{\Gamma(\sigma+1)}+\frac{1}{2 \Gamma(\sigma-1)}<2\left(3+\frac{4}{e}\right)
$$

the BVP (5)-(6) has a unique solution.

Example 3.2 Consider the BVP:

$$
\begin{gathered}
{ }^{C} D^{\sigma} \varpi(t)=3 \sin \left(\pi t+\varpi_{t}\right), \\
\varpi(0)=0, \varpi^{\prime}(0)=0, \varpi^{\prime \prime}(1)=1,
\end{gathered}
$$

where $t \in \Omega \bigcup \Lambda=[-1,1], \varpi \in C[-1,1], 2<\sigma \leq 3$. Set $f\left(t, \varpi_{t}\right)=3 \sin \left(\pi t+\varpi_{t}\right)$. We devote $\varpi_{t}(\theta)=\varpi(t+\theta)$ for each $\varpi \in C[-1,1], \quad \theta \in\left[-\frac{1}{2}, 0\right]$. Obviously, $\left(A_{2}\right)$ is satisfied. Let $M=3$. Then $\left(A_{3}\right)$ can be satisfied with $3 \sin \left(\pi t+\varpi_{t}\right) \leq 3$. Therefore by Theorem 2 , the BVP (7)-(8) has at least one solution.

Example 3.3 Consider the BVP of $\sigma$-order delay functional differential equations:

$$
\begin{gathered}
{ }^{C} D^{\sigma} \varphi(t)=\frac{\left|\varphi_{t}\right|^{2}}{\left(3+4 e^{t}\right)\left(1+\left|\varphi_{t}\right|\right)}, \\
\varphi(0)=0, \varphi^{\prime}(0)=0, \varphi^{\prime \prime}(1)=1,
\end{gathered}
$$


where $\quad t \in \Omega \bigcup_{\Lambda}=[-1,1], 2<\sigma \leq 3 . \quad$ Let $f\left(t, \varphi_{t}\right)=\frac{\left|\varphi_{t}\right|^{2}}{\left(3+4 e^{t}\right)\left(1+\left|\varphi_{t}\right|\right)}$. Obviously, $\left(A_{4}\right)$ is satisfied with $\xi(\mu)=\left(3+4 e^{-1}\right)^{-1} \mu$. In order to meet $\left(A_{5}\right)$, that is, $\quad \gamma>\frac{\zeta^{2}}{2}+\frac{\xi(\gamma) \zeta^{\sigma}}{\Gamma(\sigma+1)}+\frac{\xi(\gamma) \zeta^{\sigma}}{2 \Gamma(\sigma-1)} \quad$, we chose $\gamma>\frac{1}{2}\left[1-\left(3+4 e^{-1}\right)^{-1}\left(\frac{1}{\Gamma(\sigma+1)}+\frac{1}{2 \Gamma(\sigma-1)}\right)\right]^{-1}$, then by Theorem 3, the BVP (9)-(10) has at least one solution.

\section{Acknowledgements}

This research is supported by the Natural Science Foundation of China (617043180), and supported by Shandong Provincial Natural Science Foundation (ZR2016AM17, ZR2017MA043).

\section{References}

1. I. Podlubny, Fractional differential equations. San Diego: Academic Press, 1999.

2. Y. Zhou, Basic Theory of Fractional Differential Equations, World Scientific, Singapore, 2014.

3. M. Xu, et al., Positivity for integral boundary value problems of fractional differential equations with two nonlinear terms, J. Appl. Math. Comput., 2018 (2018) 1-13.

4. Y. Zhou, et al., Existence of nonoscillatory solutions for fractional neutral differential equations, Appl. Math. Lett., 72 (2017) 70-74.

5. A. Alsaedi, et al., Multi-term fractional-order boundary-value problems with nonlocal integral boundary conditions, Electron. J. Diff. Eq., 2018 (2018) 87. 